\title{
$\widehat{A}$ Madridge \\ madridge Journal of Nursing \\ interconnecting Scientific World
}

Research Article

Open Access

\section{Impact of CPR, AED, and Choking trainings in Nepalese Nurses}

\author{
Shanta Paudel Subedi \\ Inova Fairfax Hospital, Falls Church, VA, USA
}

\section{Article Info}

\section{*Corresponding author:}

Shanta Paudel Subedi

Instructor

American Heart Association RN

Inova Fairfax Hospital

USA

Tel: (703) 776-4001

E-mail: shantapsubedi@gmail.com

Received: May 04, 2020

Accepted: July 21, 2021

Published: July 27, 2021

Citation: Subedi SP. Impact of CPR, AED, and Choking trainings in Nepalese Nurses. Madridge J Nurs. 2021; 6(1): 180-184. doi: $10.18689 / \mathrm{mjn}-1000132$

\section{Copyright: @ 2021 The Author(s). This work is licensed under a Creative Commons Attribution 4.0 International License, which permits unrestricted use, distribution, and reproduction in any medium, provided the original work is properly cited.}

Published by Madridge Publishers

\begin{abstract}
According to The American Heart Association, cardiovascular disease is the leading cause of death worldwide. In developing countries such as Nepal, knowledge of cardiopulmonary resuscitation (CPR) for cardiac failure is rare, resulting in excessive mortality. Most cardiac arrest incidents occur outside of hospitals, and the probability of survival decreases by $7-10 \%$ for each minute CPR is delayed. Applying a "train-thetrainer" approach, the current study was conducted in Kathmandu, Nepal, where an American Heart Association-certified instructor trained nurses in CPR, automated external defibrillator (AED) use, and choking intervention. Participants' skills were measured before and after training. While the score gains were significantly greater in all three categories, the most significant gain was observed in the AED category. The findings of this study suggest that training health care workers in critical skills such as CPR, AED use, and choking intervention can greatly enhance their expertise, thereby potentially helping reduce mortality rates. This study suggests that increasing the availability of individuals with CPR skills can enhance public awareness of these interventions. Such trainings can empower Nurses to conduct similar programs, especially in developing countries such as Nepal.
\end{abstract}

Keywords: Cardiopulmonary resuscitation; Automated external defibrillator; Sudden cardiac arrest; Choking; Training.

\section{Introduction}

Heart disease is known as the number-one cause of death worldwide, according to the American Heart Association (AHA). The total number of deaths from cardiovascular disease is greater than the number of deaths from all types of cancer combined. More than 17 million people die each year from cardiovascular disease, including 655,000 in the U.S. alone [1].

One significant reason for the high number of deaths is inadequate timely help from bystanders during cardiovascular emergencies. A cardiac arrest victim's chances of survival decrease significantly if cardiopulmonary resuscitation (CPR) is not performed immediately. According to the American Heart Association CPR facts and stats, most of the cardiac arrest incidents in US occur outside the hospital setting, with $70 \%$ occurring in homes. Hence, the survival rate is only $10 \%$ for out-of-hospital cardiac arrest.

Cardiovascular disease has been recognized as a major health problem, not only in developed countries such as the U.S., but also in developing countries such as Nepal, where it accounts for $20.5 \%$ of total deaths [2]. This small, landlocked country has tremendous risk factors contributing to a high prevalence of cardiovascular disease [3]. 
Prompt CPR can double or triple a victim's chances of survival following cardiac arrest. The use of the automated external defibrillator (AED) further increases the odds of survival.

Severe choking without quick intervention leads to respiratory arrest, followed by cardiac arrest. Choking causes unintentional injury death from asphyxia. Therefore, bystander-administered CPR is extremely important to increasing the return of spontaneous blood circulation following cardiac arrest.

\section{Statement of the Problem}

Nurses are the backbone of the health care system. Hence, a top-down, "train-the-trainer" approach has significant potential for improving nursing care. Choking intervention training is simple and cost-effective, and teaching the appropriate technique can save lives. Such training is intended to bring public awareness of "Good Samaritan laws" and CPR, to increase bystanders' confidence and willingness to perform CPR. Training health care professionals in the first phase and extending training to the public in the second phase can make a significant impact. Bystanders have a pivotal role in reducing the cardiovascular mortality rate. Teaching resuscitation skills and empowering families to prepare for emergencies is critical in Nepal.

Data collection is always challenging in developing countries, especially in rural areas. Since cause of death is not always reported, Nepal's cardiovascular death rate may be even higher. Similarly, we often hear about choking incidents in the news. The high number of deaths in these cases is associated with long wait times for emergency medical services due to traffic and narrow roads in urban areas. In rural areas, lack of easy access to transportation, poor infrastructure, and inadequate resources are major challenges. Moreover, lack of routine physical checkups and insurance plans for the majority of the public leads to additional risk of heart disease and death.

Spreading awareness of disease prevention and quick intervention in cardiac arrest situations is essential. Unfortunately, CPR intervention in public is rare, further contributing to high mortality rates. Unclear Good Samaritan laws, lack of adequate professional support to nurses, and unaffordable AHA trainings contribute to the low prevalence of bystander help in Nepal. Bystanders' unwillingness to help during medical emergencies is often attributed to fear of legal repercussions.

Access to AED for public use in Nepal is poor. Also, nurses do not receive adequate CPR training from nursing schools. First aid skills such as CPR and choking intervention can be taught to anyone. Hence, bystander assistance is extremely important in areas with scarce emergency medical resources.

Motivating health care workers is an important component in reducing cardiovascular morbidity and mortality. Without educating members of the formal health care system, it is impossible to motivate the general public. Similarly, without providing training to the general public, it is impossible to reduce number of deaths from sudden cardiac arrest. A considerable amount of literature has been published in these areas. Knowledge of CPR, a basic resuscitation measure, is not adequate in the personnel connected with medical colleges in Nepal. Such knowledge is especially limited among faculties in basic science and nursing education. Knowledge of CPR was found to be correlated with having received CPR training. Fewer faculty in basic science and nursing schools with CPR training indicates their low priority [4].

Successful treatment of patients suffering cardiac arrest outside a hospital underscores a system-of-care approach to out-of-hospital cardiac arrest, which can be termed prearrival care initiated by bystanders, including recognition of cardiac arrest and seeking emergency assistance, CPR, and use of an AED. Timely pre-hospital cardiac arrest management is critical to significant survival outcomes [5].

A number of researchers have sought to identify barriers to inadequate bystander intervention in cases of cardiac arrest. The main barriers include situation awareness, communication, and attitude. A team approach, where dispatchers work as "team leaders" of callers and bystanders may potentially improve treatment of cardiac arrest victims [6]. Inadequate bystander help during emergencies is not new. Resolving the problem requires spreading awareness in the community and expanding CPR training. Based on the author's experiences working in Nepal and recent interactions with colleagues, there is still a lack of autonomy among Nepalese nurses. Despite nurses' critical role in mitigating cardiac arrest in health care settings, they are not well trained in resuscitation skills, which could build confidence and empower nurses to make critical decisions during emergencies.

The American Heart Association's training and awareness programs worldwide have resulted in tremendous increase in lives saved. The author has heard inspiring stories from bystanders trained by the author and her team in the Washington, DC metro area. The trainings have been organized voluntarily by the Society of American Nepalese Nurses (SANN) since 2017.

Inspired by the impact of bystander CPR training in the U.S., the author was motivated to implement similar trainings in Nepal. The initial phase focused on training health care professionals from both nursing colleges and hospitals. The next phase will extend the trainings to the general public. The author believes that only when health care professionals are trained can they be empowered to train community members. The training will be sustainable in the long run, with support from nursing colleges, hospitals, and communities. A topdown approach generates community awareness of training needs. The demonstration and surveys conducted during the first phase of training measured the immediate impact, and future research will assess the lifesaving impact. Continuous and sustainable training will help bring a positive and desirable outcome. As more people are trained with skills to take appropriate action, more lives can be saved. 
A large number of Nepali youths have been working in Arabic countries such as Saudi Arabia, Qatar, and others. Unfortunately, several incidents of cardiac arrest have been reported, with lack of timely help from bystanders. This illustrates how education plays an integral role in reducing the mortality rate worldwide. The COVID-19 pandemic brought additional urgency to the issue, which will be addressed in a later section.

\section{Methods}

As the first phase of training, the current study was carried out in Kathmandu, Nepal. Participants included approximately 100 bachelor's and master's level nursing students, who were divided into four groups. These participants had worked as a staff nurse for at least one year or more after graduating from Associate Degree in Nursing. An American Heart Associationcertified instructor trained these nurses in CPR, AED use, and choking intervention skills, in both English and Nepali. The contents of the training were based on American Heart Association teaching materials, delivered through explanation, slides, and video presentations. Hands-on training in CPR and AED use with manikins and choking intervention training with a choking vest were performed. Educational materials in Nepali, such as slides and the author's published articles on $\mathrm{CPR}$, defibrillator, and choking intervention were provided to each student. Data were collected anonymously, though tracking of pre and post-test results by participants, using coding. Participants were informed that the test was optional. Ninety-three participants completed both pre and post-test surveys. Participants' knowledge of CPR, AED use, and choking intervention was measured pre and post-testing, and gain scores were evaluated.

The study used the transferability aspect of Grounded Theory approach. Items measured the transferability of knowledge of CPR, AED use, and choking intervention. Such an approach motivates nurses to act not only in the clinical setting, but also in bystander roles in cardiac arrest situations. Trained nurses can also motivate other bystanders to act during emergencies. Bystanders found acting in an emergency to be disturbing, panicking, and shocking, which must be overcome [7]. Prompt action to save a cardiac arrest victim becomes difficult due to misconceptions, such as agonal breathing being misinterpreted as normal. The Grounded Theory model helped provide positive insights, build nurses' confidence, and manage their emotions.

Certain elements should be considered during CPR training to improve public perception and confidence: first, gradually increasing positive attitudes and perceptions of CPR and AED use, based on the latest scientific evidence. For example, CPR and AED use not only save lives, but also reduce fears and concerns regarding these interventions; second, promoting positive societal expectations that encourage bystander CPR and defibrillator use; and, finally, promoting a sense of empowerment and confidence that using a defibrillator is within their capability [8].

\section{Instruments}

The instrument used in this study comprised 20 multiplechoice questionnaires adopted from the American Heart Association provider manual. It included 13 questionnaires from CPR, 3 questionnaires from AED use, and 4 questionnaires from choking. The instrument measured participants' knowledge of CPR with critical understanding, such as the first action to take in cardio-respiratory emergencies; basic life support sequence of compression, airway, breathing; difference between cardiac arrest and heart attack; rate and depth of chest compression; opening airway in neck trauma victim, etc. Similarly, understanding of AED use, such as when and how to use an AED, universal AED steps, and using an AED on a victim with other devices, such as pacemaker, are AED related questionnaires. Finally, understanding of choking intervention was measured by recognizing mild airway obstruction, the technique of performing five back slaps and five chest thrusts in infants, and action to take for an unresponsive child during abdominal thrusts.

\section{Result and Discussion}

Table 1. Shows the scores from pre-test, post-test, and gain scores by participants' level of education. The results are disaggregated by the level of education in which participants are currently enrolled.

Table 1. Pre-test, Post-test, and Gain Scores by Participants' Level of Education

\begin{tabular}{|l|l|l|l|l|l|l|l|}
\hline \multirow{2}{*}{ Group } & \multirow{2}{*}{ N } & \multicolumn{2}{|l|}{ Pre-test } & \multicolumn{2}{|l|}{ Post-test } & \multicolumn{2}{l|}{ Gain Score } \\
\cline { 3 - 8 } & & Mean & Std* $^{*}$ & Mean & Std & Mean & Std \\
\hline Bachelors of Nursing & 47 & 9.57 & 2.86 & 17.49 & 1.72 & 7.91 & 2.92 \\
\hline Masters of Nursing & 46 & 11.54 & 2.28 & 18.00 & 2.04 & 6.46 & 2.45 \\
\hline Grand Total & 93 & 10.55 & 2.76 & 17.74 & 1.89 & 7.19 & 2.78 \\
\hline
\end{tabular}

*Standard deviation

One of the major findings to emerge from the study is that training health care workers with lifesaving skills is the first step in transferring such skills to the general public. Resuscitation training made a significant difference in students' level of understanding. The table shows the pretest, post-test, and gain score by participants' level of education. It shows the mean and standard deviation of all three categories by educational level. Although the gain score was higher in all three categories, the greatest gain was observed in the AED use category. Please note that, due to the difference in number of questionnaires across categories, it is prudent to compare weighted-average gain scores. The weighted gain score for each participant was computed by dividing the gain score by the number of questionnaires in the given category. The weighted average gain score for AED (0.53) was higher than that for choking intervention (0.44) and CPR (0.30). Although students at the bachelor's level had lower pretest knowledge, as expected, their gain score was greater than that of master's-level students. Knowledge 
transfer was evidenced by the meaningful gain scores.

Figure 1. Provides the graphical display of average pre and post test score by participants' education level

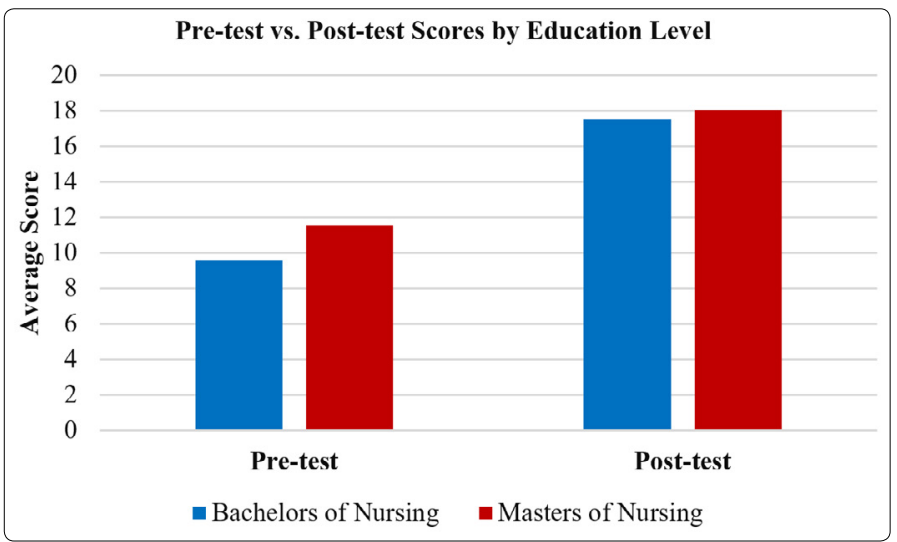

Figure 1. Pre-test vs. Post-test Scores by Educational Level

\section{Analysis of the feedback}

Participants were asked to provide feedback at the end of the training session. Most of the participants wished the training had been longer so that they could practice more, receive feedback from their own practice with manikins, and build their confidence. Also, participants wished to have regular trainings and follow-ups. Understanding the ramifications of such follow-up training, the author had planned the training, but it was paused for safety due to the COVID-19 pandemic. Trainings will resume once it is safe to carry them out in large groups.

\section{Conclusion}

The study was conducted to determine the impact of CPR training in Nepalese nurses, and it demonstrates a significant increase in nurses' knowledge and skills after training. Knowledge transfer was observed clearly during onsite demonstration and post-test questionnaires. Participants were able to retain critical knowledge during this short training. While it was not formally measured in the study, participants exhibited a high degree of motivation in elevating their knowledge and skills in critical lifesaving measures. Also, the training provided them an opportunity to build their confidence in their skills.

\section{Discussion and significance of the study}

Code situation can be stressful and demanding. Hence, practice in a controlled environment can be beneficial for staff, leading to improved patient outcomes [9]. This study has an implication for capacity building and collaboration of resource persons with CPR training and public awareness. This research will serve as a basis for future studies and motivate others to join this mission. Training health care workers with critical resuscitation skills can greatly improve their expertise, thereby reducing the cardiovascular mortality rate. Carefully developed training programs, such as those described in this study, contribute to transferring skills and empowering families to take appropriate action in emergency situations.

The author believes that long-term impact from this training is meaningful as it motivates others to initiate training on a broader scale by creating a ripple effect. Also, such training helps bridge the gap between health care professionals and the public. The study also empowers passionate nurses and other health care workers to volunteer similar training in other developing countries. The training is reliable, measurable, and outcome driven, and the impact can be seen within a short span of time. Also, schoolteachers can be trained in CPR skills as part of their professional development and further spread awareness.

There are several other groups of people, including health care workers and the public, interested in joining this training. The author believes that the training brought positive recognition to the urgency of training. Although large-group onsite training has not resumed for the public due to COVID-19, more community engagement has been seen in online Zoom trainings organized via community social media. Follow up communication shows that the community is interested in learning bystander CPR via online training until in-person classes resume. Training so far has been provided not only in English, but also in the local language (e.g., Nepali).

The author's collaboration with nursing schools and the volunteer training provided a superior example of alumni's role in bridging the gap between nursing students' training and the latest advanced health care practices worldwide. Also, it ignites health care workers globally in joining this mission.

\section{Limitation}

This study was limited to the Kathmandu valley. CPR training is critical to extending outside the Kathmandu valley because of the scarcity of health care resources. The results reported in this study are based on quantitative measures only. A follow up qualitative study was planned for 2020, but was postponed due to COVID-19. With the help of regular follow up study and extended training, the impact could be increased.

\section{COVID challenges}

While the COVID-19 pandemic was not an issue during this study, COVID guidelines need to be maintained to replicate similar studies or training. COVID-19 has created greater urgency due to the added challenges, with health care workers at significant risk of infection. Hands-only CPR is safe for cardiac arrest first aid as long as bystanders wear masks and cover victims' faces with cloth or a mask, per American Heart Association guidelines [10]. Patients are afraid to go to the hospital when they are sick due to fear of COVID. It is already too late by the time they reach the hospital in a situation such as cardiac arrest, heart attack, or stroke. American Heart Association COVID-19 resuscitation guidelines have evolved throughout the pandemic. CPR and intubation are aerosol-generating procedures; hence, it is critical to follow institutional guidelines during in-hospital cardiac arrest. Clear policies for crisis situations and contingency standards for CPR are essential so that response teams are not left in untenable situations while balancing their responsibilities [11]. 


\section{Acknowledgement}

I would like to express my appreciation to Center for Medical Simulation (CMS), Kathmandu, Nepal for their help in data collection, and to Lalitpur Nursing Campus (LNC) students for their participation.

\section{References}

1. Central for Disease Control and Prevention. Heart Disease Facts. 2021.

2. World Health Organization. World Life Expectancy. Coronary Heart Disease in Nepal. Website https://www.worldlifeexpectancy.com/nepalcoronary-heart-disease.

3. Vaidya A. Tackling cardiovascular health and disease in Nepal: epidemiology, strategies and implementation. Heart Asia. 2011; 3(1): 87-91. doi: 10.1136/heartasia-2011-010000

4. Amatya $\mathrm{M}$, Gorkhali B. Cardiopulmonary resuscitation: knowledge amongst Nepalese health personnel. Janaki Medical College Journal of Medical Science. 2016; 3(1): 25-30. doi 10.3126/jmcjms.v3i1.15372

5. Glass GF, Brady WJ. Bystander Intervention in Out-of-Hospital Cardiac Arrest. JAMA Network Open. 2019; 2(3): e191008. doi: 10.1001/ jamanetworkopen.2019.1008
6. Linderoth G, Hallas P, Lippert FK, et al. Challenges in out-of-hospital cardiac arrest - A study combining closed-circuit television (CCTV) and medical emergency calls. Resuscitation. 2015; 96: 317-322. doi: 10.1016/j.resuscitation.2015.06.003

7. Mausz J, Snobelen P, Tavares W. "Please. Don't. Die.": A Grounded Theory Study of Bystander Cardiopulmonary Resuscitation. Circ Cardiovasc Qual Outcomes. 2018; 11(2): e004035. doi: 10.1161/ CIRCOUTCOMES.117.004035

8. Liaw SY, Chew KS, Zulkarnain A, et al. Improving perception and confidence towards bystander cardiopulmonary resuscitation and public access automated external defibrillator program: how does training program help? Int J Emerg Med. 2020; 13(1): 13. doi: 10.1186/ s12245-020-00271-3

9. Williams KL, Rideout J, Pritchett-Kelly S, et al. Mock Code: A Code Blue Scenario Requested by and Developed for Registered Nurses. Cureus. 2016; 8(12): e938. doi: 10.7759/cureus.938

10. American Heart Association. Coronavirus (COVID19) Resources for CPR Training.CPR.heart.org https://cpr.heart.org/en/resources/coronaviruscovid19-resources-for-cpr-training.

11. Kramer DB, Lo B, Dickert NW. CPR in the Covid-19 Era - An Ethical Framework. N Engl J Med. 2020; 383(2): e6. doi: 10.1056/NEJMp2010758 